\title{
Estudo preliminar para a remediação de áreas rurais após um acidente nuclear ou radiológico
}

Elaine Rua Rodriguez Rochedo Diogo Neves Gomes Silva Christiano de $\mathrm{Luca}^{3}$ Pedro Rua Rodriguez Rochedo Maria Angélica Vergara Wasserman $^{4 \star}$ Daniel Vidal Perez ${ }^{5}$

${ }^{1}$ Instituto de Radioproteção e Dosimetria Av. Salvador Allende s/no Recreio do Bandeirantes Rio de Janeiro, RJ, Brasil CEP 22783-127

${ }^{2}$ Instituto de Biofísica

Universidade Federal do Rio de Janeiro Av. Carlos Chagas Filho, 373 - G1-019 llha do Fundão

Rio de Janeiro, RJ, Brasil CEP 21941-902

${ }^{3}$ COPPE - Planejamento Energético Universidade Federal do Rio de Janeiro Cidade Universitária Rio de Janeiro, RJ, Brasil CEP 21941914

${ }^{4}$ Instituto de Engenharia Nuclear - CNEN Rua Hélio de Almeida, 75

Cidade Universitária, Ilha do Fundão

Rio de Janeiro, RJ, Brasil CEP 21941-972

${ }^{5}$ Empresa Brasileira de Pesquisa Agropecuária Centro Nacional de Pesquisa de Solos Rua Jardim Botânico, 1024

Jardim Botânico

Rio de Janeiro, RJ, Brasil

CEP 22460000

* Autora correspondente

angelica.wasserman@gmail.com

\section{RESUMO}

A avaliação das medidas de proteção e de remediação em áreas rurais contaminadas por um acidente nuclear é mais complexa do que para as áreas urbanas, devido à forte influência de grande número de parâmetros e variáveis associadas a clima, dieta, hábitos alimentares, práticas agropecuárias e tipo do solo da área. Assim, não é possível realizar estudos gerais aplicáveis a qualquer tipo de área. Estudos específicos devem ser feitos nas áreas com maior probabilidade de serem contaminadas devido a um acidente nuclear. No caso da Central Nuclear brasileira em Angra dos Reis, a priorização destas áreas foi baseada em aspectos radiológicos e econômicos. Este estudo preliminar demonstrou que as diferenças nas doses de ingestão devido a diferentes tipos de solo no Brasil são mais relevantes do que as diferenças regionais nas dietas. O produto mais relevante para a dose no público foi identificado como sendo o leite. A contribuição da ingestão de leite para a dose total depende da época do ano quando o acidente ocorre. O momento de aplicação da medida de proteção tem um efeito importante na redução percentual da dose que pode ser alcançada. Em particular, para o I-131, as medidas de proteção relacionadas com a ingestão de leite devem ser consideradas dentro da fase de emergência a fim de serem eficientes.

Palavras-chave: remediação, áreas agrícolas, otimização, acidente nuclear

\section{ABSTRACT}

The assessment of protective and remediation measures for rural areas is more complex than that for urban areas, due to the strong influence of large number of parameters and variables associated with climate, diet, eating habits, farming practices and the type of soil in the area. Thus, it is not possible to perform generic studies applicable to any kind of area. Specific studies and surveys should be made in the areas most likely to be contaminated due to a nuclear accident. Considering the Brazilian Nuclear Power Central, the prioritization of areas was based on radiological and economic aspects. This preliminary study demonstrated that the different soil types in Brazil are more relevant to the ingestion dose than the regional differences in the diets. The most relevant product for public dose was identified as the ingestion of milk. The contribution of milk to total ingestion dose depends on the season of the year when the accident occurs. The timing of the application of the measure has an important effect on the percentage dose reduction that can be achieved. In particular for I-131, protective measures related to milk ingestion must be considered within the emergency phase in order to be effective.

Keywords: remediation, agricultural areas, optimization, nuclear accident. 


\section{INTRODUÇÃO}

O estabelecimento de critérios para priorizar ações de proteção e/ou remediação para áreas agrícolas é consideravelmente mais complexo do que para áreas urbanas. Diversos fatores ambientais afetam a dinâmica da concentração nos alimentos. Entre estes, podemos citar como relevantes:

- o tipo de solo presente no local das plantações e de pasto;

- a época do ano em que ocorre o acidente, devido a efeitos de sazonalidade;

- praticas agropecuárias, também dependentes de sazonalidade, tais como épocas de plantio e colheita ou práticas de alimentação de animais;

- hábitos da população, em particular a dieta, o que inclui, para grupos locais, a produção própria de alimentos no local e, para a população em geral, depende do sistema de distribuição de alimentos local, regional e até nacional, em alguns casos.

A dinâmica das concentrações nos diferentes tipos de alimentos é também extremamente
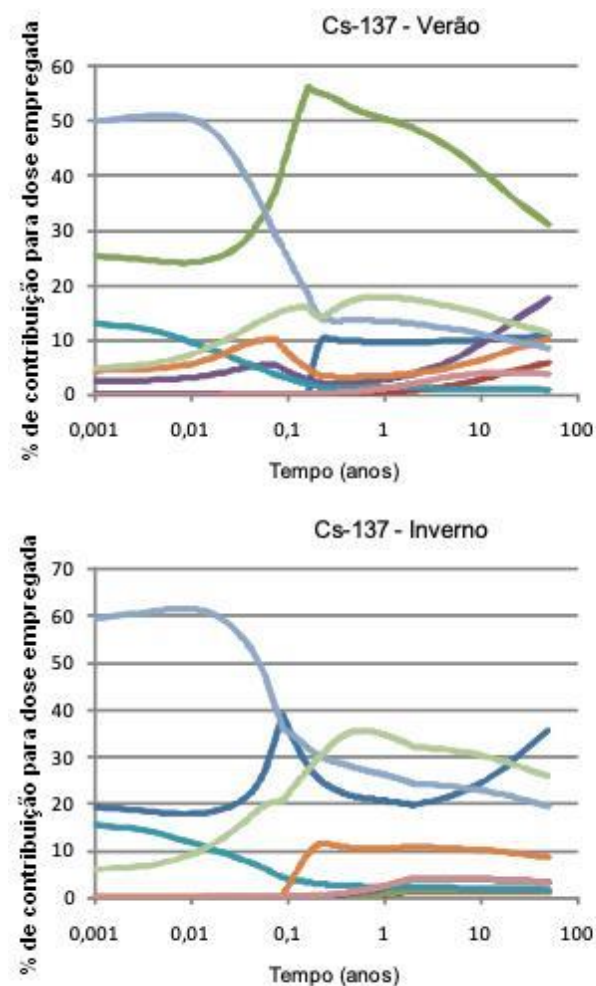

Contribuição percentual dos diferentes tipos de alimento para a dose integrada, devido a Cs-137, em função do tempo, para acidentes ocorrendo em diferentes estações do ano (Silva, 2011).

A compreensão dos mecanismos e dos processos que influenciam a transferência e a biodisponibilidade de substâncias radioativas nos ecossistemas ainda é um assunto importante para a investigação no domínio da

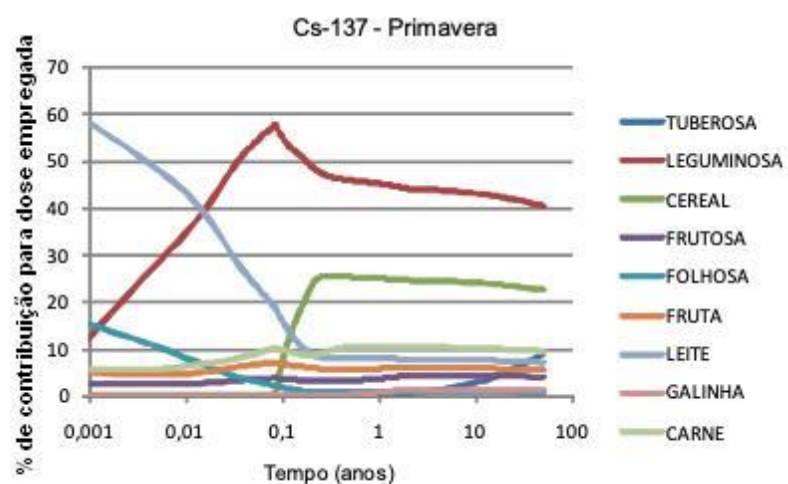

Figura 1

variada, não só de acordo com o tipo de alimento, mas também com o tempo após a contaminação (Silva 2011). A dinâmica da concentração nos principais tipos de alimento que constituem a dieta humana é demonstrada na Figura 1, para o radionuclídeo Cs-137. Pode ser observado que as concentrações nos diferentes tipos de alimentos variam com o tempo após a deposição e com o período do ano em que ocorre a deposição. Isto se deve aos diferentes estágios de crescimento dos vegetais e do pasto de acordo com a estação do ano, bem como à dinâmica do césio em cada tipo de vegetal. Considerando que cerca de $50 \%$ da dose é recebida no primeiro ano, verificamos, por exemplo, que, no caso de um acidente no verão, os alimentos que mais contribuirão para a dose são os cereais, a carne e o leite; no outono, são os cereais e os vegetais leguminosos e tuberosos; no inverno, a carne, o leite o os vegetais tuberosos; e, na primavera, as leguminosas e cereais (Silva 2011).

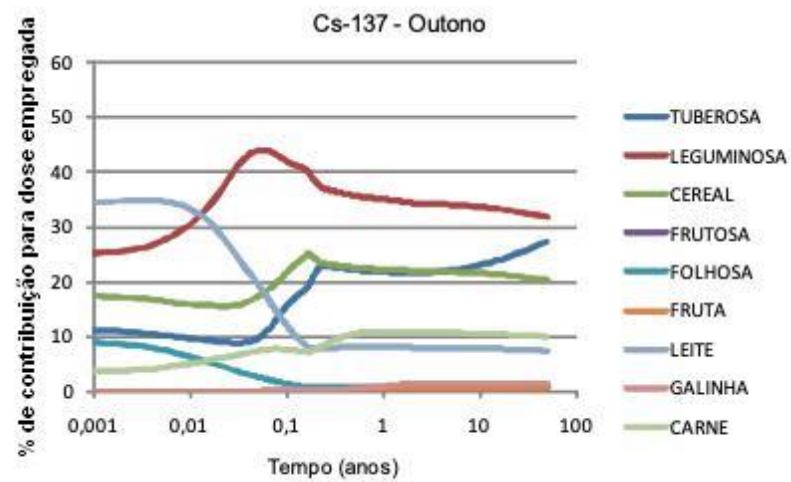

radioecologia. Estudos sobre o acidente de Chernobyl contribuíram para uma melhor compreensão do comportamento dos radionuclídeos artificiais em solos de clima temperado, fornecendo informações consisten- 
tes para a gestão de áreas rurais contaminadas e.g., Fesenko et al (2005) e IAEA (2012).

No entanto, estudos radioecológicos efetuados em zonas tropicais mostraram que alguns ecossistemas apresentam condições adequadas para uma maior absorção de radionuclídeos pelas plantas. Em alguns casos, a acidez do solo, a abundância de hidróxidos e óxidos de $\mathrm{Al}$ e $\mathrm{Fe}$, e baixo nível de fertilidade do solo foram citados como as prováveis causas da elevada transferência do Cs-137 do solo para as plantas (Wasserman 2008).

Neste contexto, os solos brasileiros são particularmente interessantes para esse tipo de estudo, já que mais de $60 \%$ deles exibem tais propriedades (Coelho et al. 2002). Sabe-se que o Latossolo e o Argissolo são predominantes no território brasileiro e, além de serem altamente intemperizados, ocupam áreas extensivamente cultivadas (Coelho et al. 2002).

Quanto aos radionuclídeos, observa-se que, de modo diverso da área urbana, vários radionuclídeos incluem o potencial de doses significativas na população (IAEA 2006). Neste

\section{METODOLOGIA}

Foram desenvolvidos diversos estudos na tentativa de avaliar os parâmetros mais relevantes para efetuar estudos específicos para áreas agrícolas. Na primeira série de estudos, foram avaliados parâmetros para o Brasil, como um todo. Nestes estudos foram considerados a relevância da dieta e a relevância do tipo de solo. A relevância do tipo de solo indicou ser este um tema prioritário a ser estudado. Posteriormente iniciamos estudos prévios para avaliar a prioridade dos municípios que fazem parte da área de planejamento estabelecida para áreas rurais, em relação a sua relevância como centro produtor agropecuário e a sua relevância em relação à dose na população, decorrente da ingestão da produção agropecuária.

A área de planejamento estabelecida para ambientes rurais foi estabelecida em estudos anteriores e compreende todos os municípios inseridos, de forma parcial ou total, em um raio de $50 \mathrm{~km} \mathrm{em}$ torno da Central Nuclear Almirante Álvaro Alberto, em Angra dos Reis, RJ (Vinhas et al, 2005). Os municípios selecionados para compor a área de estudo são:

(i) Estado de São Paulo: Arapeí, Areias, Bananal, Cunha, São José do Barreiro, Silveiras, Ubatuba; e,

(ii) Estado do Rio de Janeiro: Angra dos estudo, foram selecionados os radionuclídeos Cs-137, I-131 e Sr-90 para as avaliações sobre as consequências de contaminação de áreas agrícolas.

Simulações iniciais indicaram grande variação sazonal para a avaliação da eficiência de medidas de proteção do público (Silva 2011). Desta forma, o estudo de áreas agrícolas tornase muito complexo devido à variedade de produtos agropecuários que compões a dieta alimentar humana, diversidade de tipos de solo, diversidade de radionuclídeos relevantes e efeitos de sazonalidade, além da dinâmica do comportamento destes radionuclídeos no sistema solo-planta, após uma deposição acidental.

O objetivo principal deste trabalho foi então definir critérios básicos e utilizá-los para priorizar estudos de radioecologia e mapeamento do solos em áreas tropicais, baseado nas características de solo e produções agropecuárias das áreas no entorno da Central Nuclear Almirante Alvaro Alberto em Angra dos Reis.

Reis, Barra Mansa, Itaguaí, Mangaratiba, Parati, Piraí, Resende, Rio Claro, Volta Redonda.

Os municípios foram então avaliados em relação àqueles que seriam mais afetados por uma contaminação e sua relevância para a produção total da área de estudo, considerando o custo da perda da produção de cada município e a relevância das doses decorrentes de uma contaminação da produção local; os produtos agropecuários foram também avaliados tanto em relação ao custo de perda da produção quanto em relação à dose na população. Estas avaliações foram efetuadas de forma preliminar, considerando apenas o Cs-137 e uma população adulta.

Finalmente, foi efetuado um estudo adicional para a avaliação preliminar do efeito da dose decorrente da ingestão de leite. Neste estudo, foram considerados os efeitos na dose de adultos e crianças e os efeitos da aplicação de processamento de leite em outros produtos na dose da população. Para este estudo, foram considerados os radionuclídeos Cs-137, I-131 e Sr-90.

Os dados de hábitos alimentares foram levantados junto ao Estudo de Consumo Fami- 
liar efetuados pelo IBGE [2010a]. Os principais solos agrícolas foram levantados junto à UFLA (2013). A seleção foi feita de forma a minimizar o número de solos estudados, enquanto maximizando a área coberta do território nacional e, ainda, considerando a disponibilidade de dados específicos de fatores de transferência.

Os dados de hábitos agropecuários (épocas de plantio e colheita) e respectiva produtividade agrícola utilizados foram aqueles levantados por Vinhas et al (2005) para a região sudeste. Os fatores de transferência utilizados para $o$ Latossolo vermelho-amarelo (LVA) foram aqueles utilizados por Vinhas et al (2005). Para os demais solos, foram utilizados os valores sugeridos por Wasserman et al (2008) para cereais e, para as demais culturas, foram utilizados os fatores sugeridos por Frissel et al (2002).

Foram consideradas contaminações ambientais iniciais de $1 \mathrm{MBq} / \mathrm{m}^{2}$ de cada um dos radionuclídeos (Cs-137, I-131 e Sr-90). A comparação entre os diferentes tipos de solo foi feita só para o radionuclídeo Cs-137, devido à falta de disponibilidade de dados para os demais radionuclídeos. O modelo computacional SIEM foi utilizado para efetuar os cálculos de dose para as diferentes regiões.

Para um mesmo tipo de solo, doses individuais em áreas agrícolas, embora dependendo dos itens produzidos nos municípios, não se espera que sejam muito diferentes entre diferentes locais, devido à condição de sustentabilidade de comunidades agrícolas.

Doses coletivas, no entanto, podem ser muito diferente considerando a produtividade das áreas selecionadas. Além disso, a produção de diferentes produtos entre municípios leva a perdas de rendimento muito distintas no caso de proibição de consumo de alimentos. Assim, dois parâmetros foram considerados para criar uma ordem de classificação para priorizar áreas de pesquisa para estudos de radiovulnerabilidade: o custo da perda de produção e a dose coletiva evitada devido à

\section{RESULTADOS}

\subsection{EFEITO DA DIETA}

Para avaliar o efeito da dieta, foi considerado apenas o solo LVA (Figura 2). As diferenças entre as doses efetivas integradas em 50 anos, considerando a dieta proveniente de cada uma das regiões é de no máximo $25 \%$ para o Cs-137, proibição de alimentos um mês após o evento acidental.

Doses coletivas são restritas a itens de alimentos, enquanto valor econômico incluiu todos os produtos de cada município, incluindo cana-de-açúcar utilizada para a produção de álcool usado como combustível, produtos de origem animal e produtos de silvicultura, como o carvão, lenha e madeira em tora.

Informações sobre a produção e os preços dos produtos agropecuários foram obtidos a partir dos censos agropecuários do IBGE (2010 b). Para as estimativas de doses coletivas, o programa SIEM (Conti et al. 2002) foi usado para avaliar a concentração do alimento um mês após a ocorrência do acidente. O mesmo acidente foi simulado como acontecendo em fevereiro, maio, agosto e novembro, a fim de cobrir os aspectos de sazonalidade da dose de ingestão. A dose coletiva foi estimada multiplicando a concentração de cada alimento pela produção do município e pelo coeficiente de conversão de dose.

Para estimar resultados quantitativos decorrentes de processamento de alimentos, foram utilizados os dados compilados pela IAEA (2010). A avaliação de laticínios exige uma análise cuidadosa, devido à variedade de processos empregados e produtos gerados e dos destinos dos subprodutos de cada processamento, por exemplo, descarte como rejeito ou utilização na confecção de rações para animais.

Neste trabalho, apenas os produtos principais dos processamentos foram avaliados. Os valores para a eficiência dos procedimentos foram avaliados utilizando o software SIEM (Conti et al, 2002) para a dieta média típica da região sudeste do Brasil. As taxas de consumo per capta de alimentos processados foram obtidos a partir do IBGE (2010A). Os processamentos de leite simulados foram relativos à obtenção dos produtos leite em pó, creme de leite e leite condensado. As simulações foram efetuadas para os radionuclídeos Cs-137, I-131 e Sr-90.

$15 \%$ para o iodo e $30 \%$ para o estrôncio. A maior diferença observada em relação ao $\mathrm{Sr}-90$ é devido ao maior consumo de leite observado na região sul do país. 


\subsection{EFEITO DO TIPO DE SOLO}

Considerando toda a dieta de cada região sendo proveniente de cada tipo de solo (Latossolo Vermelho- Amarelo, Argissolo e Latossolo vermelho não férrico), para o Cs-137, observou-se que as doses integradas em 50 anos devidas a Latossolo Vermelho não férrico são bastante superiores àquelas devidas aos demais tipos de solo, sendo a menor dose relativa ao Argissolo, para todas as dietas consideradas. As diferenças percentuais entre as doses relacionadas ao Latossolo Vermelho não férrico e Argissolo, em relação às doses relacionadas ao Latossolo Vermelho Amarelo estão apresentadas na Tabela 1. Pode ser observado que o tipo de solo pode ter uma influência de até cerca de $70 \%$, sendo, portanto, maior do que a diferença observada devido a diferentes tipos de dieta observadas no país.

Tabela 1 - Diferença percentual das doses obtidas para diferentes tipos de solo, Latossolo Vermelho não férrico (LV) e Argissolo (A), em relação àquela relativa a um solo tipo Latossolo Vermelho-Amarelo (\%).

\begin{tabular}{lcccc}
\multirow{2}{*}{ Região } & \multicolumn{2}{c}{ Dose no primeiro ano } & \multicolumn{2}{c}{ Dose na vida inteira (50 anos) } \\
\cline { 2 - 5 } & LV & A & LV & A \\
\hline NORTE & 2,5 & $-0,53$ & 49 & $-8,9$ \\
NORDESTE & 3,7 & $-0,40$ & 64 & $-7,6$ \\
SUDESTE & 3,7 & $-0,52$ & 61 & -10 \\
SUL & 3,0 & $-0,58$ & 53 & -11 \\
CENTRO-OESTE & 3,7 & $-0,53$ & 61 & -10 \\
\hline BRASIL & 3,4 & $-0,53$ & 59 & $-9,4$ \\
\hline
\end{tabular}

\subsection{PRIORIZAÇÃO DE MUNICÍPIOS E PRODUTOS}

Fator 1, perda de renda devido ao descarte de produtos: Considerando a perda financeira do descarte de produtos para toda a área de estudo, os cinco produtos mais relevantes são o leite de vaca, banana, madeira em toras, cana de açúcar e coco. A partir desses produtos, só o leite de vaca é um item relevante para a dieta da população; consumos individuais de bananas e coco são muito mais baixos. No entanto, para a produção de municípios, a perda de rendimento de qualquer um destes produtos seria significativa. Considerando-se os valores absolutos de toda a produção de cada município, a maior perda de rendimento será de Cunha (SP), seguido por Rio Claro (RJ) e Piraí (RJ).

Fator 2, doses coletivas de ingestão de produtos agropecuários: As doses de ingestão dependem da época do ano, quando ocorre a contaminação. Para avaliar a dose coletiva, foi considerada uma deposição de $1 \mathrm{kBq} / \mathrm{m}^{2}$ de Cs137 em toda a área. As doses coletivas foram estimadas e aplicadas às produções municipais. Foram consideradas as doses do primeiro ano após a contaminação.

Devido à alta produção e aos hábitos de áreas típicas de clima tropical, onde foi considerado que os animais são alimentados por pasto fresco durante todo o ano, o leite foi $o$ principal produto que contribui para doses coletivas em todas as estações.

O consumo de carne e de frango foi então avaliado, de forma preliminar. Como não há informação da produção de carne, foi considerado o consumo médio per capta da região sudeste multiplicado pelo número de habitantes de cada município, limitado pela disponibilidade de animais no município (isto é, considerando o consumo máximo de $1 / 3$ do número de cabeças). A relevância relativa dos municípios, em relação a produtos agropecuários, não foi alterada pela inclusão de carnes na dieta da população local. Em relação à dose coletiva, a ingestão de frangos tem efeito desprezível e a de carne de boi teria uma contribuição máxima em torno de $8 \%$ para acidentes ocorrendo na primavera ou no verão, $10 \%$ para um acidente ocorrendo no outono e 12 $\%$ para um acidente ocorrendo no inverno.

Como consequência, a ordem de classificação para municípios segue de perto a produção de leite anual. Os cinco municípios incluídos no estudo, que lidam com maiores valores de dose devido à ingestão de seus produtos agrícolas, são Cunha, Barra Mansa, Resende, Rio Claro e Bananal. Mais uma vez, o município de Cunha lidera a ordem de classificação para os municípios. 


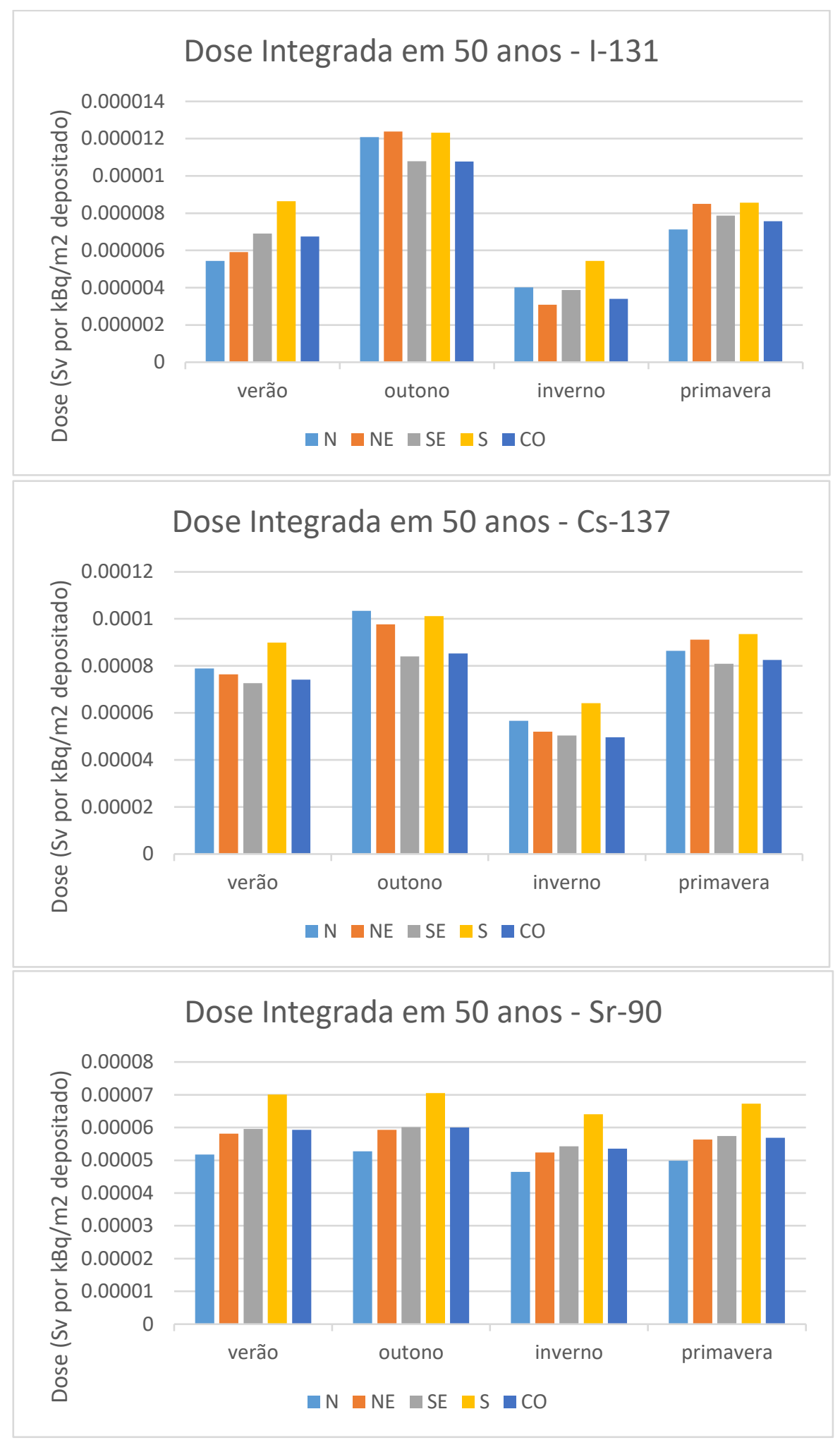

Figura 2 diferentes épocas do ano, para o Cs-137

Conclui-se então que os principais produtos para a realização de estudos radioecológicos que levem a um melhor conhecimento sobre o comportamento a longo prazo de radionuclídeos em solos de clima tropical seria o leite de vaca, seguido de frutas (principalmente de banana) e mandioca.
Combinando ambos os fatores, isto é, o custo da produção e as doses coletivas de ingestão da produção de cada município, Cunha seria o município mais relevante para o desenvolvimento de estudos radioecológicos sobre a caracterização dos solos e fatores de transferência solo-planta e, em relação aos 
produtos agropecuários, a principal via a ser estudada seria ar-solo-pasto- leite. Depois de Cunha, Barra Mansa e Resende mostraram os

\subsection{LEITE}

Considerando a relevância do leite para as doses de ingestão para acidentes ocorrendo em todas as estações do ano, alguns estudos adicionais preliminares foram então realizados. $\mathrm{O}$ primeiro estudo foi voltado para avaliar a dose em crianças. Foi considerada uma ingestão diária de $0,8 \mathrm{~L}$ de leite para crianças. Para adultos, foi considerada a dieta definida na Tabela 2 (IBGE 2010 a).

A Figura 2 apresenta os percentuais de redução da dose integrada do primeiro ano considerando diferentes momentos de introdução da remoção de leite da dieta, considerando um acidente ocorrendo na primavera. Para crianças (1 ano) foi considerada valores de classificação mais elevadas entre os municípios estudados (Rochedo et al. 2015).

apenas a ingestão de leite. Para adultos, a redução representa a redução observada em relação à dieta total.

Pode ser observado que o efeito desta medida de proteção é muito dependente do momento em que a remoção de consumo é efetuada. Em particular para crianças pequenas, é essencial que seja introduzida o mais cedo possível para ser efetiva, principalmente em relação ao I-131. Para estas, é essencial providenciar alimentação alternativa em menos de uma semana após a liberação acidental, muitas vezes ainda dentro da fase inicial da emergência radiológica que deu origem ao evento de contaminação ambiental.

\begin{tabular}{clcc} 
Tabela 2 - Dieta para adultos utilizada no estudo de ingestão de leite. & & \\
\hline & Item & Taxa de ingestão (g/d) & \\
\hline \multirow{4}{*}{ Tipo } & Cereal e grãos & 118 & \\
& Vegetais & 8 & 323 \\
& Tubérculos e raízes & 47 & \\
& Outros vegetais & 150 & \multirow{2}{*}{103} \\
\hline \multirow{2}{*}{ Carne } & Boi & 66 & 164 \\
\hline Leite & Frango & 37 & 164 \\
\hline
\end{tabular}

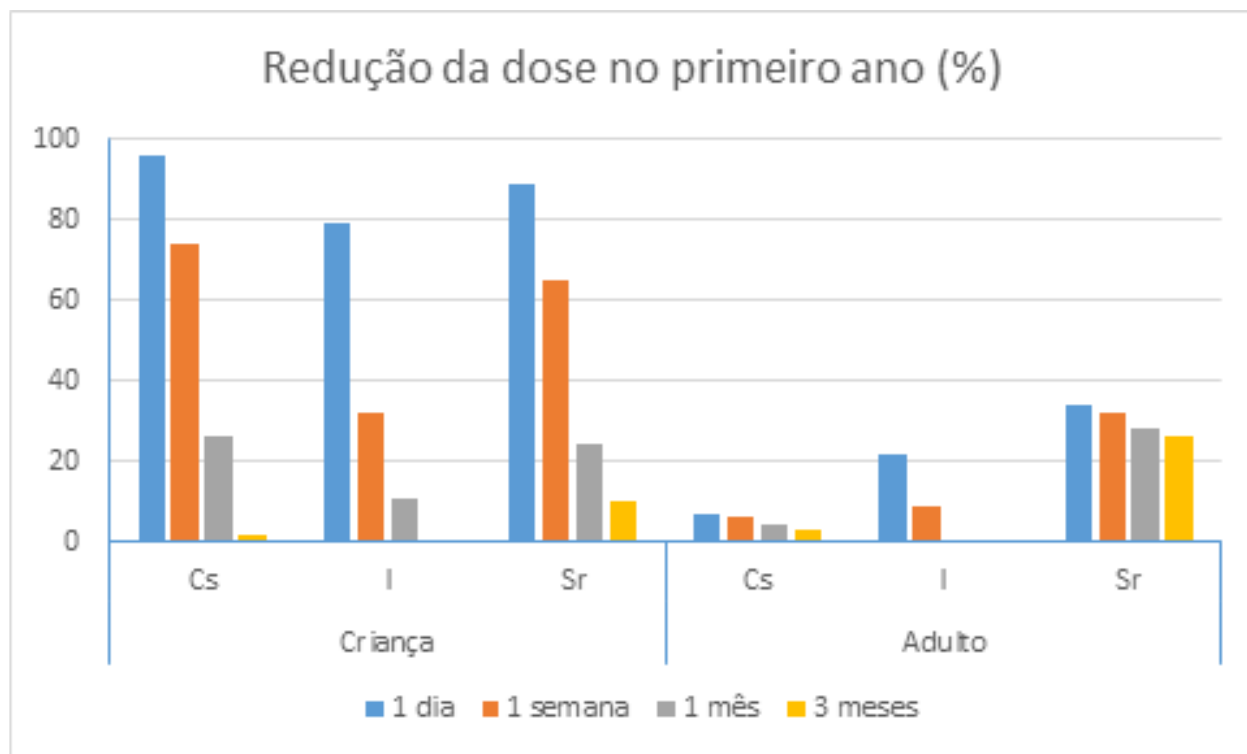

Percentual de redução da dose do primeiro ano devido à ingestão com a retirada de consumo de leite da dieta, em diversos momentos após a contaminação inicial (1 dia, 7 dias, 30 dias e 90 dias).

A relevância do leite para a economia dos municípios, porém, levou à necessidade de se tentar encontrar uma solução que evitasse o descarte do leito como rejeito, não só por seu 
valor econômico como pelo volume de material a ser descartado e também pela sua relevância para a dieta humana. Assim, foi feita uma avaliação, também de forma preliminar e genérica, das principais possibilidades de processamento do leite em outros produtos, de forma a tentar avaliar a viabilidade de aproveitamento do material retirado da dieta.

Resultados para alguns procedimentos de transformação de leite são apresentados na tabela 3. A tabela inclui a redução da dose total ingestão devido à remoção e descarte de leite da dieta. Ele também inclui o efeito do uso de leite para produção de produtos derivados e os efeitos na dose total ingestão. Para a retirada do leite da dieta, o momento em que a introdução de alimentos processados é feita, em todos os casos, mostraram forte influência sazonal nas doses.
Para CS-137, o aumento da dose total devido à introdução de produtos lácteos processados é menor do que $2 \%$ em todos os casos para a dose do primeiro ano e inferior a $1 \%$ para a dose de vida. Para I-131, como todas as doses são recebidos dentro do primeiro ano, os aumentos em doses integrados são os mesmos para o primeiro ano e vida inteira. $\mathrm{O}$ efeito de remoção de leite da dieta pode variar até $56 \%$ da dose total, enquanto que o aumento da dose devido aos produtos lácteos processados aumenta até $5 \%$ da dose total de ingestão. Para Sr-90, o efeito da remoção de leite da dieta pode variar até $67 \%$ da dose total, enquanto o aumento da dose, devido aos produtos lácteos transformados são de no máximo $5,5 \%$ da dose total ingestão (Tabela 3).

Tabela 3 - Efeito do processamento e da retirada do leite da dieta em doses ao público.

\begin{tabular}{|c|c|c|c|c|c|c|}
\hline \multirow{2}{*}{ Nuclídeo } & \multirow{2}{*}{$\begin{array}{c}\text { Estação do } \\
\text { ano } \\
\text { (acidente) }\end{array}$} & \multicolumn{2}{|c|}{$\begin{array}{c}\text { Redução de dose devido à } \\
\text { retirada do leite da dieta }(\%)\end{array}$} & \multicolumn{3}{|c|}{$\begin{array}{c}\text { Aumento da dose do primeiro ano devido à } \\
\text { introdução de produtos processados (\%) }\end{array}$} \\
\hline & & primeiro ano & vida inteira & manteiga & $\begin{array}{c}\text { leite } \\
\text { condensado }\end{array}$ & leite em pó \\
\hline \multirow{4}{*}{ Cs-137 } & Verão & 10 & 7 & 0,01 & 0,41 & 0,84 \\
\hline & Outono & 7 & 6 & 0,01 & 0,28 & 0,58 \\
\hline & Inverno & 23 & 10 & 0,01 & 0,93 & 1,93 \\
\hline & Primavera & 15 & 8 & 0,01 & 0,59 & 1,22 \\
\hline \multirow{4}{*}{ I-131 } & Verão & 32 & 32 & 0,09 & 1,28 & 2,65 \\
\hline & Outono & 20 & 20 & 0,05 & 0,80 & 1,66 \\
\hline & Inverno & 56 & 56 & 0,15 & 2,24 & 4,64 \\
\hline & Primavera & 30 & 30 & 0,08 & 1,18 & 2,45 \\
\hline \multirow{4}{*}{ Sr-90 } & Verão & 39 & 30 & 0,03 & 1,56 & 3,24 \\
\hline & Outono & 37 & 29 & 0,03 & 1,47 & 3,04 \\
\hline & Inverno & 67 & 33 & 0,05 & 2,68 & 5,55 \\
\hline & Primavera & 57 & 32 & 0,05 & 2,29 & 4,74 \\
\hline
\end{tabular}

Para todos os radionuclídeos, o processo mais eficiente em relação à dose para o público, é a produção de manteiga, ao passo que o processo menos eficiente é a produção de leite em pó. No entanto, esta considera a taxa de ingestão habitual para estes produtos e não como substitutos para o leite fresco. Além disso,

\section{CONCLUSÕES}

As decisões sobre contramedidas relacionadas com áreas agrícolas são muito mais complexas do que aquelas para uma contaminação de área urbana. Há uma forte influência da estação do ano nas doses recebidas devido à ingestão de produtos de origem vegetal e animal, a partir de uma área específica. Além disso, o tipo de solo tem uma forte influência deve notar-se que o leite em pó também pode ser armazenado durante 6 meses ou mais, o que reduziria ainda mais a dose quando da introdução do alimento no mercado. Praticamente todo o I-131 já terá decaído neste período. sobre a transferência de radionuclídeos depositados no solo para as plantas.

Este estudo demonstrou que os diferentes tipos de solo do Brasil podem afetar mais a dose de ingestão do que as diferenças nas dietas regionais. No caso de acidentes, a preocupação maior é sempre com o consumo local das áreas afetadas e, portanto, áreas em que a população tem um alto índice de auto abastecimento, como 
é o caso das regiões agrícolas. É também importante considerar quais itens da dieta são oriundos de áreas não afetadas para não restringir desnecessariamente o consumo de alimentos produzidos no local afetado pelo acidente.

Considerando os municípios dentro de um raio de $50 \mathrm{~km}$ das usinas nucleares brasileiras, sugerimos desenvolver estudos radioecológicos com prioridade para leite, bananas e mandioca nos municípios de Cunha (SP), Barra Mansa (RJ) e Resende (RJ).

O leite é o principal produto a ser avaliado em relação a doses para o público, em particular crianças. A possibilidade de utilizar $\mathrm{o}$

\section{REFERÊNCIAS}

Coelho, M.R., Santos, H.G., Silva, E.F., Aglio, M.L.D., 2002, O recurso natural solo, In: Manzatto, C.V., Junior, E.F., Peres, J.R.R. (eds.), Uso agrícola dos solos brasileiros, 1 ed., capítulo 2, Rio de Janeiro, Embrapa Solos.

Conti L.F.C., Rochedo E.R.R., Amaral E.C.S., 2002. Desenvolvimento de um sistema integrado para avaliação de impacto radiológico ambiental em situações de emergência, Rev. Bras. de P\&D, 4(3) p. 872-879.

Fesenko, S.V., Voigt, G., Spiridonov, S.I., Gontarenko, I.A., 2005. Decision making framework for application of forest countermeasures in the long term after the Chernobyl accident, J. Env. Radioact. 82: 143 166

Frissel, M.J., D.L. Debb, M. Fathony, Y.M. Lind, A.S. Mollahe, N.T. Ng, I. Othman, W.L. Robison, V. Skarlou-Alexiou, S. Topcuoglu, J.R. Twining, S. Uchida, M.A. Wasserman, 2002. Generic values for soil-to-plant transfer factors of radiocesium. Journal of Environmental Radioactivity 58:113-128

IAEA - INTERNATIONAL ATOMIC ENERGY AGENCY 2006. Environmental consequences of the Chernobyl accident and their remediation: twenty years of experience, In: Report of the Chernobyl Forum Expert Group 'Environment', Radiological Assessment Reports Series, International Atomic Energy Agency, Vienna.

IAEA - INTERNATIONAL ATOMIC ENERGY AGENCY 2010. Handbook of parameter values for the prediction of radionuclide transfer in terrestrial and freshwater environments. IAEA Technical Reports Series No. 472.Vienna,194 p.

IAEA - INTERNATIONAL ATOMIC ENERGY AGENCY 2012. Guidelines For Remediation Strategies To Reduce The Radiological Consequences Of Environmental Contamination. Fesenko S. \& Howard B.J. (eds.) Technical Report Series N.475, IAEA,Viena, 183 p

IBGE - INSTITUTO BRASILEIRO DE GEOGRAFIA E ESTATÍSTICA 2010 a. processamento em produtos derivados parece promissora, porém tem que ser avaliada também em relação aos resíduos gerados e às possíveis utilizações como, por exemplo, o uso em rações animais.

Aspectos sazonais são relevantes para as decisões sobre o processamento de alimentos após uma contaminação acidental. A ação principal na redução da ingestão de doses é a remoção de alimentos da dieta, fornecendo comida limpa para a população. Todavia, o descarte dos produtos pode ser caro, não só devido à perda da produção, mas também devido às grandes quantidades de resíduos a serem eliminados.

Pesquisa de Orçamentos Familiares 2008-2009. Instituto Brasileiro de Geografia e Estatística, Rio de Janeiro.

IBGE - INSTITUTO BRASILEIRO DE GEOGRAFIA E ESTATÍSTICA 2010 b) Produção pecuária municipal. Disponível em www.ibge.gov.br. Acessado em maio de 2012.

IBGE - INSTITUTO BRASILEIRO DE GEOGRAFIA E ESTATÍSTICA 2012. Produção agrícola municipal. Disponível em www.ibge.gov.br. Acessado em maio de 2012.

Rochedo, E.R.R., Igreja E., Silva D.N.G, Wasserman M.A.V., Barboza A.E. Priority of areas for agricultural countermeasure assessment. IRPA 2015, Buenos Aires

Silva D.N.G. 2011. Estudo da aplicação de medidas de proteção para o público e de remediação de áreas contaminadas em caso de acidentes nucleares e/ou radiológicos no Brasil. Dissertação de mestrado. IRD/CNEN, Rio de Janeiro, $106 \mathrm{p}$.

UFLA - UNIVERSIDADE FEDERAL DE LAVRAS. "Solos do cerrado". http://www.dcs.ufla.br/Cerrados/Portugues/CInt roP.htm, Acessado em janeiro de 2013.

Vinhas, D.M., Rochedo, E.R.R., Wasserman, M.A.V., Conti, L.F.C., 2005. Modeling the dynamics of radionuclide concentration in food after an accident in tropical areas. Rev. Bras. de P\& D, 7:139-144.

Wasserman, M.A., F. Bartoly, A.G. Viana, M.M. Silva, E.R.R. Rochedo, D.V. Perez, C.C. Conti, 2008. Soil to plant transfer of ${ }^{137} \mathrm{Cs}$ and ${ }^{60} \mathrm{Co}$ in Ferralsol, Nitisol and Acrisol. J. of Environmental Radioactivity 99: 546-553. 